\title{
Multi-logic Rulesets based Junction-point Movement Controller Framework for Traffic Streamlining in Smart Cities
}

\author{
Sreelatha $\mathrm{R}^{1}$ \\ ISE Department \\ B M S College of Engineering \\ Basavanagudi, Bangalore 560091
}

\author{
Roopalakshmi $\mathrm{R}^{2}$ \\ CSE Department \\ Manipal Institute of Technology \\ Manipal 576104
}

\begin{abstract}
In the internet era, Intelligent Transportation System (ITS) for smart cities is gaining tremendous attention since it offers intelligent smart services for traffic monitoring and management with the help of different technologies such as microelectronics, sensors and IoT. However, in the existing literature, very few attempts are made towards effective traffic monitoring at road junctions in terms of providing faster decision making so that the traffic present in heavily congested urban environments can be dynamically rerouted. In order to tackle this issue, this article proposes a new Controller framework that can be applied at junction-points in order to the control the traffic movement. Specifically, the proposed framework utilizes a multi-logic ruleset database to estimate the traffic density dynamically at the first stage followed by the usage of signal-time computation algorithm at the second stage in order to streamline the traffic and achieve faster clearance at the junction-points. The experimental results conducted with the help of test environment using MEMSIC nodes clearly demonstrate the improved efficiency of the proposed framework in terms various performance metrics including move command frequency, ruleset score and fluctuation score.
\end{abstract}

Keywords-Intelligent transportation systems; junction-point traffic monitoring; ruleset database; traffic density estimation

\section{INTRODUCTION}

Nowadays, Intelligent Transportation System (ITS) for smart cities is gaining huge attention, which offers smart as well as intelligent services towards traffic management and monitoring with the help of technologies including electronic sensing, data communication, and advanced information management systems [1],[2]. Further, the transportation efficiency can be significantly enhanced with the aid of the proximity cooperation of the user over the road and vehicle targeting towards reducing the congestion of the traffic [3]. Precisely, the prime idea of ITS is to address the all sorts of problems associated with traffic density over the network of the road [4]. In general, ITS system consists of the different sophisticated components such as Advanced Traffic Information Service System (ATIS), Emergency Rescue System (ERS), Freight Management System (FMS), Electronic Public Transport System (EPTS), Advanced Public Transportation System (APTS), Advanced Vehicle Control System (AVCS) and Advanced Traffic Management System (ATMs) and so on [5].

Though enormous amount of research efforts are made towards ITS research domain, yet several serious issues of traffic density control are however remaining unresolved till now in almost all the major cities in every part of the world. In addition to that, an increase in traffic density gives rise to severe congestion problems and induces pollution and thereby adversely affects economic losses. In the current scenario, the successful deployment of ITS for facilitating effective traffic monitoring is significantly affected by these two limitations: a) To standardize the elementary factors in order to compute and predict the state of deadlock condition of traffic over larger traffic density and b) To ensure the reliability associated with the predictive concept in traffic management along with higher accuracy [6]. The former limitation is addressed up to a certain extent, in the existing literature by means of Vehicular Ad-hoc Networks using forecasting approaches [7]. The latter issue is addressed using infra-structure based approaches by employing GPS data [8]. However, the state-of-the art techniques in both the cases, fail to provide effective performance, due to problems such as inaccurate estimates of traffic, delay and inadequate accuracy in dynamic and large-scale urban traffic environment.

From setup perspective, the existing literature towards ITS traffic monitoring can be broadly classified into two categories namely, infrastructure Vs non-infrastructure-based techniques. The infrastructure-based techniques primarily focus on essential functionalities such as traffic light management, route suggestion, congestion detection, re-routing and speed adjustment whereas non-infrastructure-based approaches primarily concentrate on cooperative congestion identification, congestion avoidance accident detection and warning. However, one of the significant research challenges in the existing literature is, the complexity in design framework due to which only normal classification over test environments can be performed. Further, the traffic density computations are assessed using certain formulated theories without suitable validations with real-world traffic systems, which results in significantly poor performances [9],[10]. Due to these aspects, promising techniques are needed for traffic monitoring in ITS which can effectively make faster decisions for rerouting traffic in heavily congested urban environments. In this article, a novel controller design framework is proposed, which can be deployed at junction-points in order perform effective traffic monitoring and management by formulating a unique multilogic ruleset. The organization of the article is described as follow: Section 2 discusses the existing literature, Section 3 describes the proposed along with detailed discussion of 
system design as well as algorithm. Section 4 details result analysis followed by the illustration of conclusion in Section 5.

\section{RELATED WORK}

In the existing literature, a lot of research attempts are made towards incorporating novel features in ITS so that different sorts of traffic monitoring issues can be addressing. For instance, Calabuig et al. [11] presented a novel techniques which is responsible for managing alert message for the remotely positioned vehicle by means of utilizing cluster-head selection strategies of VANETs in ITS. However, it suffers due to huge requirement of resources for unicast communication as well as non-availability of potential broadcasting features incellular networks. Also, recently, Derrouz et al. [12] developed a vehicle type classification framework using both the 3D parameters and 2D features in order to to control traffic as well as violations of road code.Specifically, 3D parameters are used to reconstruct complete dimension of given vehicle in terms of height, width and length whereas 2D features are utilized for dimensionality reduction. Though the proposed classification system can accurately detect vehicles from different view angles, yet the performance may get seriously affected due to the errors calibration process used for estimating $3 \mathrm{D}$ parameters.

Recently in 2019, Blazek et al. [13] addressed the communication latency problem by presenting a burst-error performance modelling, which utilizes the formulation of the maximum likelihood function to measure the various extensive records based on channel-based parameters. Though, the authors successfully employed the fading information from the target channel and signal noise to investigate the degree of burstiness, yet few of fault-tolerance aspects such as burstiness for stress testing may degrade the performance of the system.

Very recently in 2020, Chavhan et al. [14] proposed a context-aware public transport system based on IoT, which uses both the context information and emergent intelligence, in order to enable public transport services in metropolitan areas. Though, the proposed system provides better results, yet it fails to address towards security issues. Javed et al. [15] addressed the security problems in ITS by developing a mechanism that can detect outliers and un-authenticate the false data and thereby enhance the decision of the traffic. A study towards safety standards in ITS is carried out by Naufal et al. [16], where a control and supervision system is designed for autonomously transport systems. Dotoli et al. [17] introduced have used multi-objective optimization framework in order to perform routing of vehicles by evaluating the user's preference. A similar kind of case study is considered by the adoption of blockchain technology in ITS by the authors in [18]. Szymanski et al. [19] presented a vehicle positioning framework using spatio-temporal features of the delay caused in public transportation systems with the help of GPS. The work carried out by Zhao et al. [20] includes a probabilistic framework in order to study the pattern of flows of passengers over different trains and routes. Calvillo et al. [21] have investigated connecting bridges between all the connected devices in smart cities. The study hypothesizes on distributed energy system, which plays an important role in performance improvement in smart cities.
Recently, Tian et al. [22] developed a recurrent attention framework for identifying the traffic signs by making use of deep neural networks, which suffers due to its computational complexity. Cai et al. [23] introduced a vector-based query processing system, which carries out query processing of ITS. Very recently in 2020, Choy et al. [24] proposed a low power speed monitoring framework with the help of radio frequency as well as neural network architectures. However, the performance of the proposed framework primarily depends on the estimation accuracy and antenna design. Rafter et al. [25] developed an adaptive traffic signal system with multiple modes that integrates position data of linked vehicles with data extracted from a network in a decentralized manner over a single intersection of the road.

To summarize, in the existing literature, huge efforts are made towards introducing the usage of sophisticated mechanisms to perform traffic monitoring, which are not much explored towards compatibility issues. Specifically, every vehicle's practical scenario over the road is given least preference, which on the other way plays a vital role in the realistic traffic situations. More specifically, in most of the existing traffic monitoring systems fail to include the practical constraints such as bi-directional traffic, routing viewpoint and directionality and so on in their decision making process, which may result in slightly less realistic estimations. In addition to that, in existing literature, very few attempts are carried out towards embedding automated decision-making or intelligence within the system to perform traffic monitoring. On the other hand, the adoption of the Global Positioning System (GPS) and sensors are widely popular in the existing literature for controlling navigation and traffic monitoring. However, GPS suffers from outages many times, and they are not always accurate. Usage of the sensor is a good idea, but the sensors are used in a very simplified manner which cannot capture dynamic information about the mobility of the vehicles based on direction in a two-lane system. Furthermore, generally the bottleneck condition of the traffic density is witnessed in intersection points or junctions. When one junction has multiple routes connected, it becomes the most challenging scenario to provide the command for vehicle clearance by executing different commands of the signal light. Unfortunately, most of the existing traffic monitoring systems perform slightly less effective due to manual process in traffic monitoring. Based on these aspects, promising traffic monitoring systems are needed, which can carry out faster decision-making as well as streamlining the traffic based on dynamic traffic events over the peak density conditions on the road.

\section{A. Motivation and Contributions}

In this article, a novel Junction-point Movement Controller framework, named as JMC Framework is presented, which makes use of multi-logic ruleset database, in order to estimate the traffic density dynamically and also perform faster clearance at the junction-points. More precisely, the main contributions of the proposed JMC framework are given by,

* Construction of a new Ruleset Database consisting of multi-value logic rulesets, which are used to generate aggregated traffic density inferences in the form of vehicle statistics and thereby effectively control the traffic movement. 
* Introducing a novel Traffic Density Estimation and Signal-time Computation Algorithm, named as TDMSC Algorithm, in order to achieve consistent traffic density estimation followed by effective path determination, so that faster clearance of the vehicles can be ensured at the specified junction-points.

The proposed JMC framework along with construction of ruleset database and TDM-SC algorithm along with the detailed design is illustrated in Section 3.

\section{PROPOSED FRAMEWORK}

Fig. 1 describes the overview of proposed JMC framework architecture consists of two stages. In the first stage, the number of inbound and outbound vehicles over the specific route are first inputted into the system. Precisely, the inbound and outbound vehicles are tracked with the help of sensors, which are placed on both the entry as well as exit points of routes, assuming that the multi-routes meet at the junction points. In the second stage, the entire aggregated vehicle movement data is then passed to a controller system hosted over the junction point. The controller processes the aggregated vehicular information and applies a multi-value logic system where different possibilities of ruleset are constructed based on traffic density. The resultant information helps the controller node to understand the degree of traffic density in different routes meeting at the junction. Based on the inferences derived from the ruleset, the controller decides for clearing the vehicles from their routes, by providing three different forms of commands including MOVE command, WAIT FOR command and STOP command, whereas MOVE command plays a vital role in clearing the traffic. In this way, the proposed system provides a solution in order to handle the variable traffic density on different routes by means of introducing a faster decision-making system for facilitating traffic monitoring and management in the urban environment. The complete system design including the analytical modelling of the proposed framework is detailed in the forthcoming section.

\section{SySTEM DESIGN}

In this section, the main concept of the proposed JMC framework design is illustrated in detail, which in turn can be fully automated to optimize the traffic system, especially for any level of traffic density, so that smart traffic monitoring and controlling can be achieved. Specifically, system design modules including assumptions considered, Ruleset Database Formation followed by algorithm design are illustrated.

\section{A. Assumptions}

The implementation of the proposed JMC system is carried out by considering the following assumptions given by:

- Each vehicle V1 is equipped with a sensing device, which is having the unique identity of the vehicle.

- Every entry and exit points of a road are equipped with a transceiver which performs identification of the vehicles and aggregates the information about several inbound and outbound vehicles.

- A controller system with specific traffic commands tc is assumed to be present in every junction point

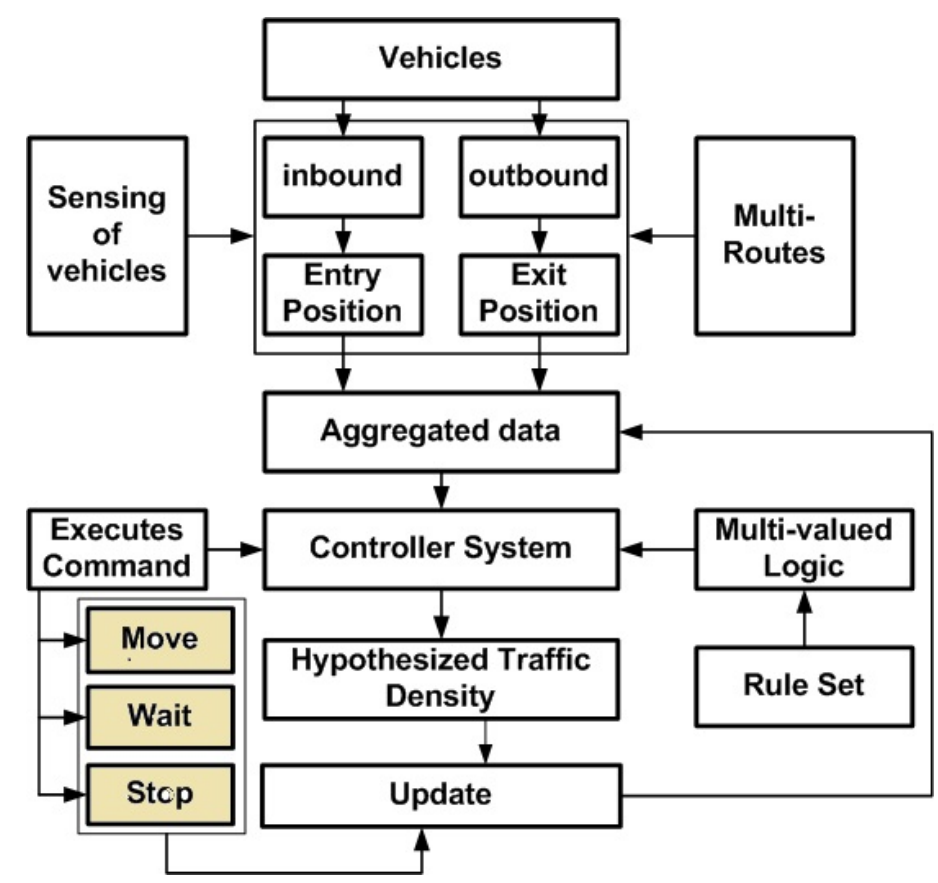

Fig. 1. Overview of the Proposed JMC Framework.

which performs execution of the ruleset in order to implement the automated and intelligent traffic management in the urban region.

- Apart from this, the proposed JMC design also assumes that, any street with a higher capacity is capable of introducing larger number of vehicles within it.

- The proposed framework also assumes that all the sensory devices are powered with a battery of finite lifetime.

\section{B. Formation of Ruleset Database}

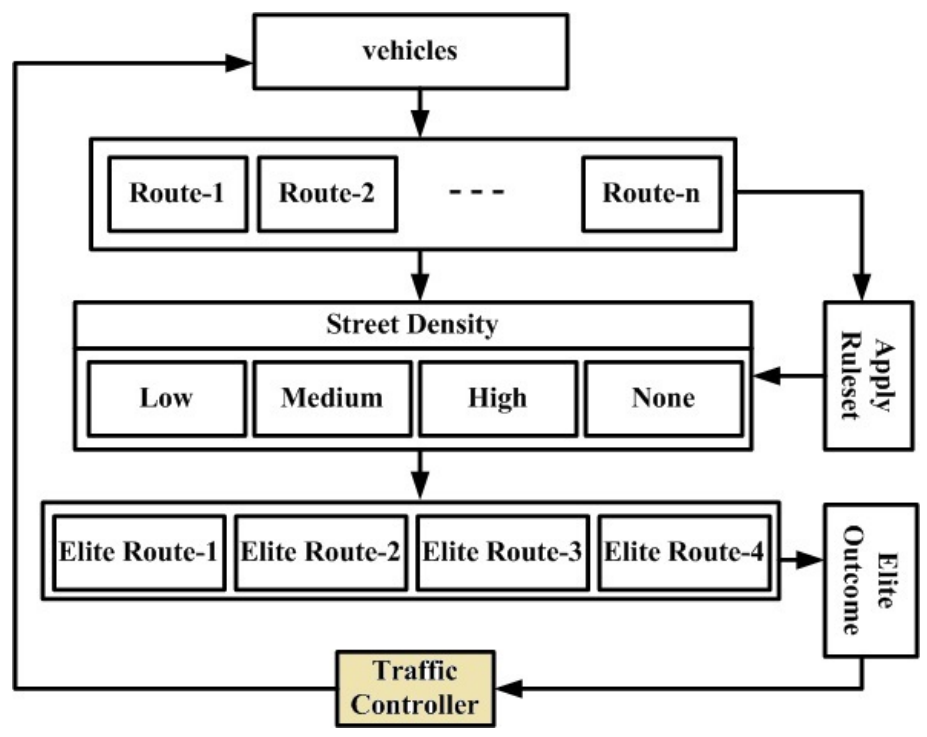

Fig. 2. Formation of Ruleset Database.

The proposed JMC framework, utilizes a Ruleset Database 
using which it controls the movement of the traffic by means of employing multi-values logic systems in the form of vehicle statistics from four sample streets. Precisely, Fig. 2 describes the concept of Ruleset Database formation based on the traffic density over the street.More precisely, in the first step, the proposed system uses two primary logical operators, namely, "and" and "then" in order to checks the density of all streets and thereby derives the inferences about the traffic density as shown in the top portion of figure. Specifically, a simplified version of a rule in the given ruleset database is given as follows:

\section{IF $(\operatorname{cond} 1) \&(\operatorname{cond} 2) \&(\operatorname{Cond} 3) \&($ Cond 4$)--T H E N--\operatorname{Inf} 1$}

In the above rule expression, cond 1 to Cond 4 represent four different street conditions corresponding to its respective traffic density in terms of low, medium, and high traffic. More specifically, cond1 includes \{low,medium,high $\}$ which represents various combination of street 1 traffic to be low, medium, and high. The similar type of rules are constructed for all combinations of traffic conditions. The inference is the outcome of the combination of these conditions resulting in selection of specific direction of a vehicle to move by choosing an outbound street, and thereby the inferences represent the outbound street. Precisely, in the proposed JMC framework, a complete Ruleset Database consisting of 80+ combinations of rulesets is constructed in order to generate the aggregated traffic density inferences and a snapshot of this database is shown in Fig. 3. After this step, the resultant traffic density

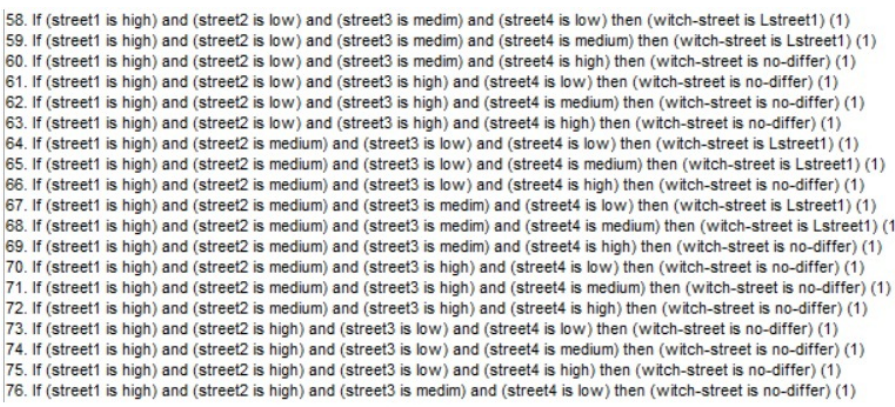

Fig. 3. Snapshot of Ruleset Database.

inferences are fed to the controller, so that it can understand the current traffic data aggregation. Followed by this step, the controller uses similar ruleset to compute the result of go time algorithm based on the traffic density and thereby performs decision-making.

\section{Algorithm Design}

The proposed JMC framework presents an algorithm named as, Traffic Density Estimation and Signal-time Computation Algorithm (TDM-SC), which is in short indicated as TDM-SC algorithm for optimizing the traffic density monitoring efficiency in the urban traffic environment. Specifically, in the proposed JMC framework, first traffic density information is calculated with the help of execution of rules in Ruleset database followed by the classification of the traffic density. Then the resultant density estimations are further employed by the Controller in order to compute signal passing time allowed for every vehicle in the specified route. More specifically, the proposed TDM-SC algorithmic with step-by-step procedure is detailed as given below.

Input: V (vehicles), tc (traffic command), iv (inbound vehicle) Output: $\alpha$ (execution of traffic command)

Start

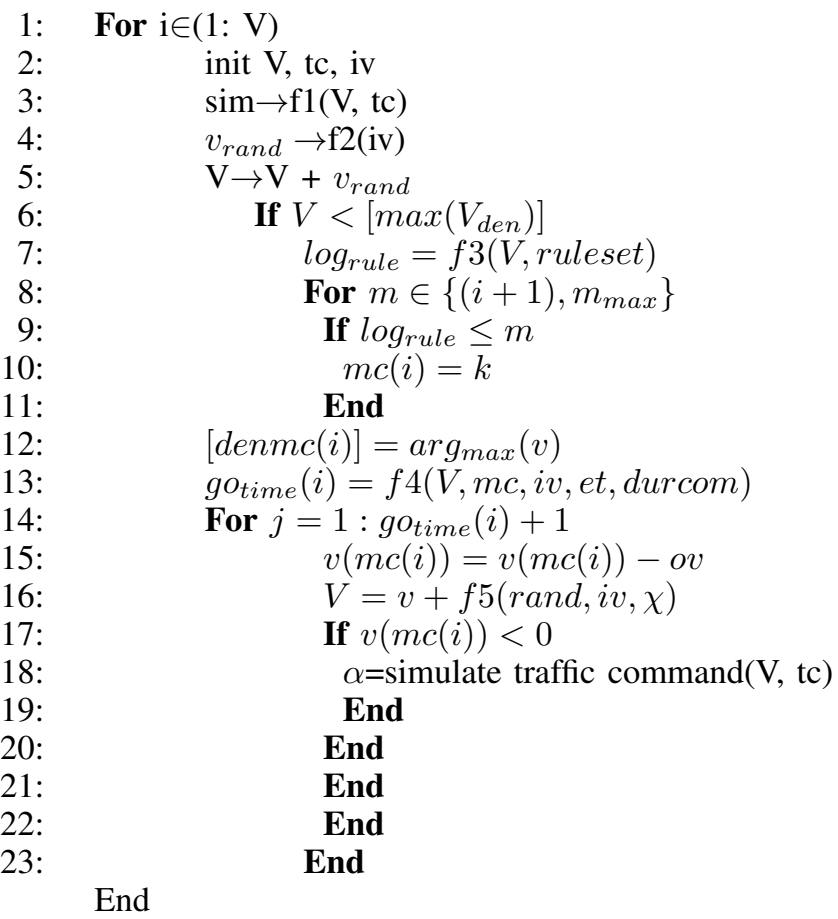

a) The working of TDM-SC algorithm is illustrated as follows:: The first initialization step of TDM-SC algorithm is to turn on the controller system for computation of the vehicle's signal-time calculations. Then, the proposed algorithm takes the inputs namely, input of $\mathrm{V}$ (vehicles), tc (traffic command), and iv (inbound vehicle), which after processing yields an outcome of $\alpha$ (execution of traffic command). Precisely, the algorithm initially considers all the vehicular nodes $\mathrm{v}$ as specified in step-1 of Fig. 4 (Line-1). The variable tc (traffic control) represents a controller system that directs the vehicle's movement using standard signal lights. The variable $i v$ and $o v$ represent the total number of vehicles sensed by sensors over the streets entry and exit points respectively. The proposed study considers four entry points that meet at the junction where the controller system resides. An explicit function $\mathrm{f} 1(\mathrm{x})$ is implemented which is responsible for simulating the vehicles $\mathrm{V}$ and traffic command tc over the junction point as shown in step-3(Line-3). The proposed system considers the junction point as a prominent bottleneck condition of routing within the junction itself in the urban traffic system.

The next part of the implementation focuses on generating the random number of vehicles $v_{\text {rand }}$ by considering the function $\mathrm{f} 2(\mathrm{x})$, which in turn takes a random number of incoming vehicles iv in step-4 (Line-4). Therefore, the total number of vehicles on each route is the addition of vehicles $\mathrm{V}$ and a random number of vehicle $v_{\text {rand }}$ as specified in step5 (Line-5). In order to depict a realistic traffic scenario, the proposed algorithm takes a fixed number of maximum vehicle density $v_{d e n}$ on all of four considered routes as given in step6 (Line-6). A logical ruleset $\log _{\text {rule }}$ is computed by applying a function $\mathrm{f} 3(\mathrm{x})$ considering the input argument of vehicles 
$\mathrm{V}$ and ruleset created earlier as specified in step-7 (Line-7). After this step, the function $\mathrm{f} 3(\mathrm{x})$ performs the logical ruleset's evaluation by considering the four membership functions of 4 constituent routes meeting at the junction point.

Depending on the deployed environment of vehicular movement on the four test routes, the algorithm compares the value of the ruleset $\log _{\text {rule }}$ with the $m$ variable, representing the output degree of density of the road as shown in step-8 (Line-8). Depending on the degree of road density encountered $m$, the algorithm assigns $k$ to the move command $m c$ as given in step-9(Line-9).It should be noted that $k<<m$, which means that if the vehicles density is found higher, the number of move command towards every 4 points of streets, is $\mathrm{k}=1$, $2,3,4$ (Line-10). For example, if the value of the $\log _{\text {rule }}$ is found less than 3 , it will signal move command $m c$ to 1 st route $(\mathrm{k}=1)$. If the value of $\log _{\text {rule }}$ is found less than 6 , it will signal move command $m c$ to 2 nd route $(\mathrm{k}=2)$. Similarly, if $\log _{\text {rule }}$ is found less than 9 , it signals move command $m c$ to 3 rd route $(\mathrm{k}=3)$, and so on as shown in Fig. 4.

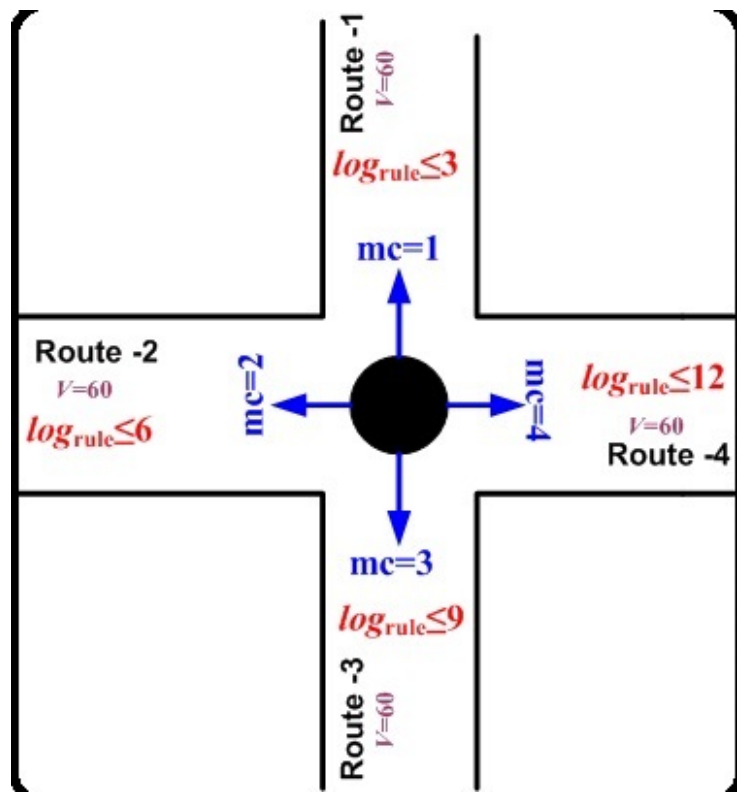

Fig. 4. Signal-Time Computation for Directing Vehicles Through Signals.

\section{Result AND Discussion}

\section{A. Experimental Setup:}

The experimental setup of the proposed JMC framework including the test environment, deployment scenario as well as simulation nodes are detailed in this section as follows. Precisely, the deployment scenario including the urban traffic for the proposed JMC framework is shown in Fig. 5, in which the simulation environment consisting of inbound and outbound vehicles are visible on the assigned routes connected to respective junction points. More precisely, simulations are carried out with the help of MATLAB and the primary details considered in this setup are illustrated as follow:

- Urban traffic scenario is created by the deployment of Junction-points, that are constructed with 4 orthogonally joined routes.
- All the routes have both inbound and outbound vehicles in terms of 2-lane system with bidirectional traffic is considered.

- The entry and exit point are simulated with standard MEMSIC nodes which are responsible for estimating both inbound and outbound vehicles. Specifically, with one MEMSIC node in each route, the setup is implemented by 4 MEMSIC nodes which can capture from 5 to up to 3000 vehicles in entire simulation rounds.

- The junction point is equipped with the proposed centralized JMC system, which accepts the aggregated data from all the routes and performs the traffic density estimation followed by the signal-time computations by making use of TDM-SC algorithm, as described in the previous section in order to control the traffic density.

- Further, the duration of individual outbound cars is considered as 0.3 seconds. Also, the average number of inbound cars are set as 2 whereas for outbound cars it is 12 .

- The simulations are carried out from a lower bound of 50 iterations to 500 iterations, in order to test the efficiency of the proposed framework.

- The simulation is also carried out for sample traffic conditions, where same number of vehicles are allocated in all streets at same time. For an example, all streets have 60 vehicles to be cleared in one signal. This sample scenario is also considered and proposed framework utilizes suitable rulesets in order to make appropriate decisions.

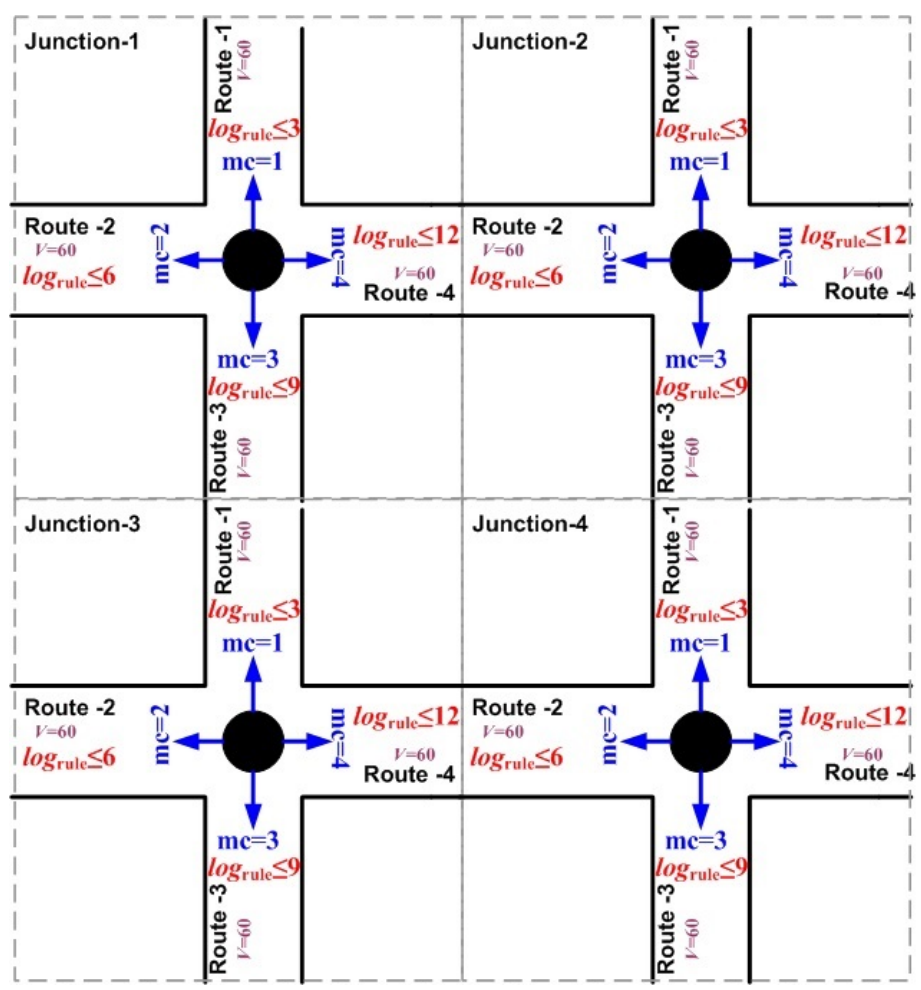

Fig. 5. Simulation Environment for Proposed JMC Framework. 


\section{B. Results and Discussion}

The performance of the proposed JMC framework is evaluated in terms of analyzing different parameters, such as no. of vehicles passed and effectiveness multi-value logic set and so on. More specifically, the efficiency of the proposed framework is demonstrated in terms of analyzing the four important parameters as given by,

a) Analysis of Frequency of MOVE Command and number of vehicles passed.

b) Analysis of Fluctuation Score.

c) Analysis of Ruleset score and

d) Analysis of Command Transition duration.

1) Analysis of MOVE Command Frequency and number of vehicles passed: Fig. 6(a) and (b) show the simulation results of move command frequency as well as vehicles passed in terms of bar graphs with respect to the corresponding routes, which are inbound to junction points. Precisely, move command frequency is defined as the number of times the controller executes signal for moving the vehicles in order to facilitate clearance to move, which is similar to green light signal. The simulations are carried out up to 500 iterations, in which it exhibits increasing occurrence of vehicle clearance for all four sample routes as shown in Fig. 6(a). Similarly, a number of vehicles that are allowed to pass also exhibit an incremental curve as indicated in Fig. 6(b), which in turn proves the efficiency of the proposed framework.
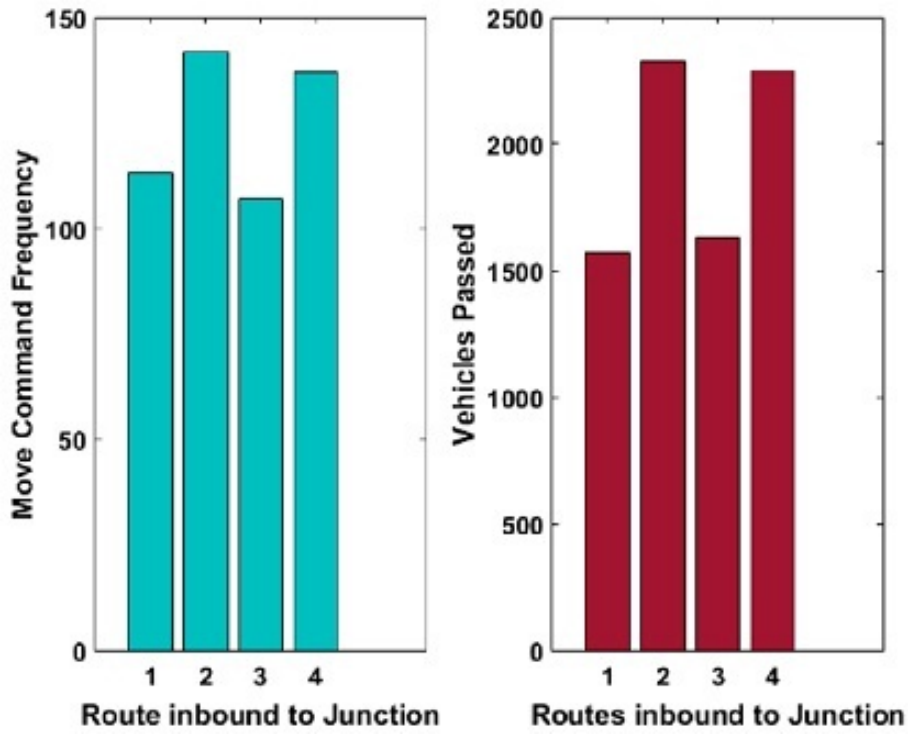

Fig. 6. (a) Analysis of Move Command Frequency (b) No. of Vehicle Passed.

2) Analysis of Fluctuation Score: Fig. 7 shows the results of second performance parameter -fluctuation score, which is a dimensionless unit, for a maximum of 500 simulation rounds. Specifically, Fluctuation score is defined as, the degree of execution of the logical ruleset considering three scenarios including lower, medium and higher traffic along with the time for move command is computed. More specifically, the goal is to find differences in the duration of the move command which is needed for vehicle clearance in every defined route. Using ruleset, if the time duration of the move command is found lesser than 2 seconds or 5 seconds or more than 5 seconds, then the system automatically allocates scores of value of $0.8,0.9$, and 1 respectively in order to calculate the rate of fluctuation. Fig. 7 clearly indicates that, though different fluctuation rates are shown in the various routes inbound to the junction points, however the fluctuation score is restricted within the maximum of 1-2 units only. In this way, Fig. 7 demonstrates the improved consistency of the proposed JMC framework in terms of lower degree of fluctuations, by means of balancing the complete traffic system without any occurrences of deadlock situations.

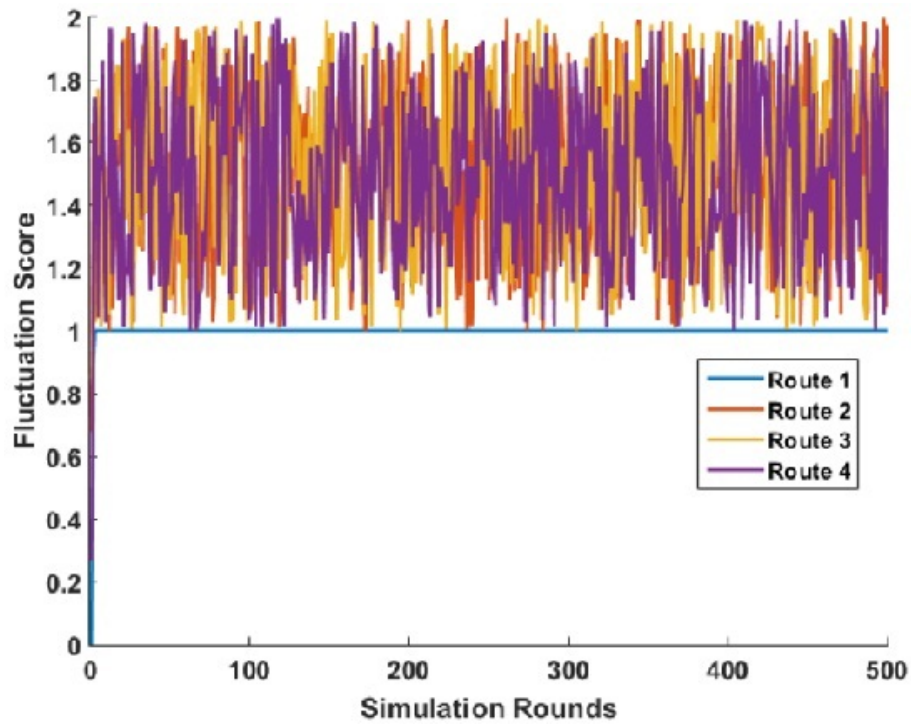

Fig. 7. Analysis of Fluctuation Score.

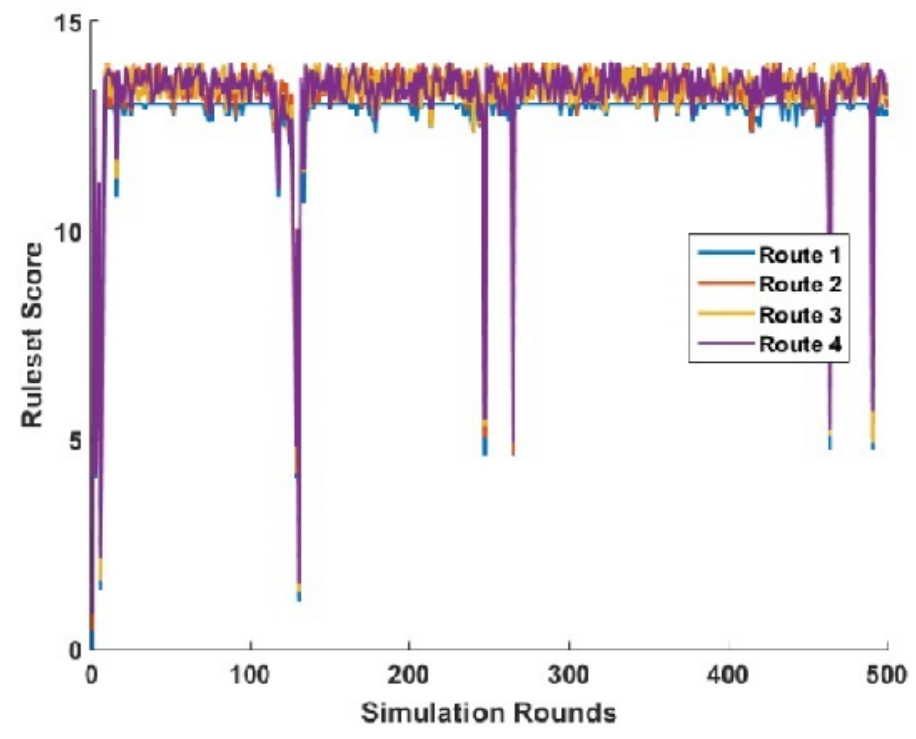

Fig. 8. Analysis of Ruleset Score.

3) Analysis of Ruleset score: Fig. 8 illustrates the results of Ruleset score, which is an essential parameter, since it plays a significant role in predicting performance of the proposed JMC framework. Precisely, ruleset score responsible for finding out the actual need of the ruleset on 4 test routes. It is to be noted that, in the proposed system, ruleset database consisting of 81 


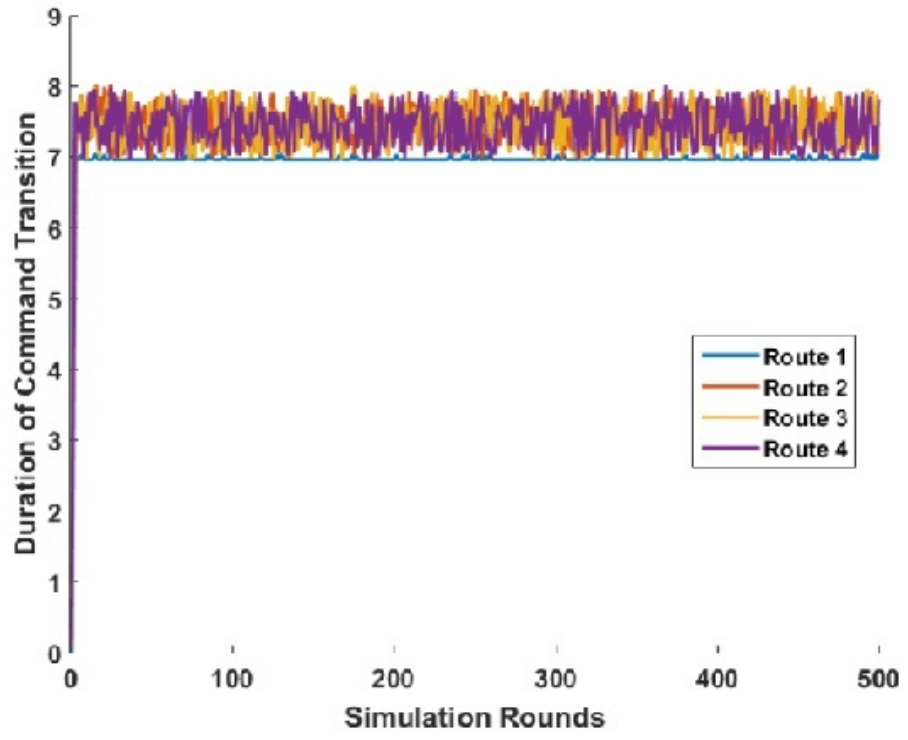

Fig. 9. Analysis of Duration of Command Transition.

rulesets covering all possible cases of traffic density is utilized as described in Section 3. Fig. 8 graph indicates that the rulesets are capable of dynamically controlling the variable rate of traffic density with higher consistency for all four sample routes. It is also observed in Fig. 8 that, though there is an increase in number of simulation rounds, yet there is not much significant divergences in the ruleset scores. Therefore, Fig. 8 results demonstrate the better consistency of ruleset scores and thereby prove the scalability feature support of the proposed JMC framework, even in case of heavy traffic environments.

4) Analysis of Duration of Command Transition: Fig. 9. highlights the result graphs of fourth performance parameter duration of command transition, in terms of simulation rounds vs command transition durations. Precisely, this parameter specifies the duration of command release performed by a controller with respect to move, stop, and wait for signals. From Fig. 9, it can be observed that, a consistent outcome is indicated for all the respective routes, which in turn demonstrates the efficiency of the proposed system in monitoring and managing the traffic system. Though, the number of inbound entries over the junction increase, yet a consistent performance is indicated in the Fig. 9 graphs, which clearly proves the efficiency of the proposed framework.

\section{CONCLUSION}

In this article, a novel junction-point movement controller framework is presented, which can efficiently perform traffic monitoring and management by means of employing multilogic rulesets. Precisely, the significant features of the proposed system are: i) usage of non-complex mechanism to carry out decision making for the elite route to be selected, ii) inclusion of routes connected at junction point with faster clearance of the vehicles, iii) system capable of counting the both inbound and outbound vehicles and thereby effective path determination and traffic density estimation becomes easier, iv) usage of the non-iterative process to offer cost-effective solution.In future, the proposed framework can be enhanced for inclusion of security features, adaptive properties and so on, so that it can be reasonably applied in real-world urban traffic situations.

\section{ACKNOWLEDGMENT}

The authors would like to thank the anonymous reviewers for their valuable feedback and suggestions.

\section{REFERENCES}

[1] Okarma, Krzysztof, Darius Andriukaitis, and Reza Malekian, Sensors in Intelligent Transportation Systems Hindawi Journal of Advanced Transportation, 1-2, 2019.

[2] Guerrero-Ibáñez, Juan, Sherali Zeadally, and Juan Contreras-Castillo, Sensor technologies for intelligent transportation systems MDPI Journal of Sensors 18, no. $4,1212-1224,2018$.

[3] Roozemond, D. A., Intelligent traffic management and urban traffic control based on autonomous objects Sixth annual conference on AIS'96, Artificial intelligence, simulation, and planning in high autonomy systems, pp. 23-37. 1996.

[4] Benalla, M., B. Achchab, and H. Hrimech, Improving driver assistance in intelligent transportation systems: An agent-based evidential reasoning approach Journal of Advanced Transportation,vol.20,1-4,2020.

[5] Ben-Akiva, M, Intelligent Transportation Systems (ITS) and the Impact of Traveler Information \& Emerging Themes in Transportation Economics and Policy Transportation Systems Analysis: Demand \& Economics,USA, 2008.

[6] Sładkowski, Aleksander, and Wiesław Pamuła, eds, Intelligent transportation systems-problems and perspectives Springer international publishing, Vol. 303, 2016.

[7] Hadiwardoyo, S.A., Patra, S., Calafate, C.T. et al. An Intelligent Transportation System Application for Smartphones Based on Vehicle Position Advertising and Route Sharing in Vehicular Ad-Hoc Networks Journal. Comput. Sci. Technol. 33, 249-262,2018.

[8] Salazar-Cabrera, Ricardo, Álvaro Pachón de la Cruz, and Juan Manuel Madrid Molina. Sustainable transit vehicle tracking service, using intelligent transportation system services and emerging communication technologies: a review Journal of Traffic and Transportation Engineering, 1-19,2020.

[9] Qureshi, Kashif Naseer, and Abdul Hanan Abdullah, A survey on intelligent transportation systems Middle-East Journal of Scientific Research 15, no. 5, 629-642,2019.

[10] Sumalee, Agachai, and Hung Wai Ho, Smarter and more connected: Future intelligent transportation system Iatss Research 42, no. 2,6771,2018 .

[11] D. Calabuig, D. Martín-Sacristán, J. F. Monserrat, M. Botsov and D. Gozálvez, Distribution of Road Hazard Warning Messages to Distant Vehicles in Intelligent Transport Systems in IEEE Transactions on Intelligent Transportation Systems, vol. 19, no. 4, pp. 1152-1165, 2018, doi: 10.1109/TITS.2017.2718103.

[12] H. Derrouz, A. Elbouziady, H. Ait Abdelali, R. Oulad Haj Thami, S. El Fkihi, and F. Bourzeix, Moroccan Video Intelligent Transport System: Vehicle Type Classification Based on Three-Dimensional and Two-Dimensional Features IEEE Access, vol. 7, pp. 72528-72537, 2019, doi: 10.1109/ACCESS.2019.2920740.

[13] T. Blazek and C. F. Mecklenbräuker, Measurement-Based Burst-Error Performance Modeling for Cooperative Intelligent Transport Systems IEEE Transactions on Intelligent Transportation Systems, vol. 20, no. 1, pp. 162-171, Jan. 2019, doi: 10.1109/TITS.2018.2803266.

[14] S. Chavhan, D. Gupta, B. N. Chandana, A. Khanna and J. J. P. C. Rodrigues, IoT-Based Context-Aware Intelligent Public Transport System in a Metropolitan Area IEEE Internet of Things Journal, vol. 7, no. 7, pp. 6023-6034, July 2020, doi: 10.1109/JIOT.2019.2955102.

[15] M. A. Javed et al., ODPV: An Efficient Protocol to Mitigate Data Integrity Attacks in Intelligent Transport Systems IEEE Access, vol. 8, pp. 114733-114740, 2020, doi: 10.1109/ACCESS.2020.3004444.

[16] J. K. Naufal et al., A2CPS: A Vehicle-Centric Safety Conceptual Framework for Autonomous Transport Systems IEEE Transactions on Intelligent Transportation Systems, vol. 19, no. 6, pp. 1925-1939, June 2018, doi: 10.1109/TITS.2017.2745678. 
[17] M. Dotoli, H. Zgaya, C. Russo and S. Hammadi, A Multi-Agent Advanced Traveler Information System for Optimal Trip Planning in a Co-Modal Framework IEEE Transactions on Intelligent Transportation Systems, vol. 18, no. 9, pp. 2397-2412, Sept. 2017, doi: 10.1109/TITS.2016.2645278.

[18] A. Lei, H. Cruickshank, Y. Cao, P. Asuquo, C. P. A. Ogah, and Z. Sun, Blockchain-Based Dynamic Key Management for Heterogeneous Intelligent Transportation Systems IEEE Internet of Things Journal, vol. 4, no. 6, pp. 1832-1843, Dec. 2017, doi: 10.1109/JIOT.2017.2740569.

[19] P. Szymański, M. Żołnieruk, P. Oleszczyk, I. Gisterek and T. Kajdanowicz, Spatio-Temporal Profiling of Public Transport Delays Based on Large-Scale Vehicle Positioning Data From GPS in Wroctaw IEEE Transactions on Intelligent Transportation Systems, vol. 19, no. 11, pp. 3652-3661, 2018, doi: 10.1109/TITS.2018.2852845.

[20] J. Zhao et al., Estimation of Passenger Route Choice Pattern Using Smart Card Data for Complex Metro Systems IEEE Transactions on Intelligent Transportation Systems, vol. 18, no. 4, pp. 790-801, April 2017, doi: 10.1109/TITS.2016.2587864.

[21] C. F. Calvillo, Á. Sánchez-Miralles and J. Villar, Synergies of Electric
Urban Transport Systems and Distributed Energy Resources in Smart Cities IEEE Transactions on Intelligent Transportation Systems, vol. 19, no. 8, pp. 2445-2453, Aug. 2018, doi: 10.1109/TITS.2017.2750401.

[22] Y. Tian, J. Gelernter, X. Wang, J. Li, and Y. Yu, Traffic Sign Detection Using a Multi-Scale Recurrent Attention Network IEEE Transactions on Intelligent Transportation Systems, vol. 20, no. 12, pp. 4466-4475, Dec. 2019, doi: 10.1109/TITS.2018.2886283.

[23] Z. Cai, F. Ren, J. Chen and Z. Ding,Vector-Based Trajectory Storage and Query for Intelligent Transport System IEEE Transactions on Intelligent Transportation Systems, vol. 19, no. 5, pp. 1508-1519, May 2018, doi: 10.1109/TITS.2017.2726103.

[24] J. L. C. Choy, J. Wu, C. Long and Y. Lin,Ubiquitous and Low Power Vehicles Speed Monitoring for Intelligent Transport Systems IEEE Sensors Journal, vol. 20, no. 11, pp. 5656-5665, 2020, doi: 10.1109/JSEN.2020.2974829.

[25] C. B. Rafter, B. Anvari, S. Box and T. Cherrett, Augmenting Traffic Signal Control Systems for Urban Road Networks With Connected Vehicles IEEE Transactions on Intelligent Transportation Systems, vol. 21, no. 4, pp. 1728-1740, 2020, doi: 10.1109/TITS.2020.2971540. 